\title{
Relación de severidad de los deterioros de los pavimentos flexibles con la transitabilidad
}

\section{Relationship of severity of deterioration of flexible pavements with walkability}

\section{RESUMEN}

Abner Itamar León Bobadilla

\begin{abstract}
Objetivo: Determinar la severidad de los deterioros superficiales de los pavimentos flexibles y su relación con la transitabilidad. hipótesis: Existe relación entre la severidad de los pavimentos flexibles con la transitabilidad, en nivel de significación ( $P>0,05)$. Dado que Chi cuadrado: 23,87 $>=9.48$. Existe relación entre la severidad de deterioros de pavimentos flexibles con la transitabilidad. Metodología: Es una investigación aplicada, no experimental, descriptivo, correlacional, transversal con enfoque cualitativo; en una población de 150 calles de Nuevo Chimbote. Se utilizó el método de evaluación de deterioros superficiales en pavimentos flexibles y su relación con la transitabilidad, según Resolución Directoral № 022018-MTC/14. en una muestra de 108; se utilizó la prueba de chi cuadrado para determinar la asociación entre los deterioros superficiales en pavimentos y la transitabilidad. Existe una relación entre la severidad de los deterioros superficiales y transitabilidad en los pavimentos flexibles en un nivel de significancia de $(P>0,05)$. Conclusiones: El deterioro de los pavimentos flexible arroja un $25 \%$ de buena transitabilidad, un $40 \%$ en regular transitabilidad y un $35 \%$ en mala transitabilidad. Existe relación entre los deterioros de los pavimentos flexibles con la transitabilidad.
\end{abstract}

Palabras claves: Deterioro de pavimentos flexibles; Transitabilidad. Severidad de deterioro de pavimento flexible.

\section{ABSTRACT}

Objective: To determine the severity of the surface deterioration of flexible pavements and its relationship with passability. Hypothesis: There is a relationship between the severity of flexible pavements and walkability, at a significance level $(P>0.05)$. Since Chi square: $23,87>=9,48$. There is a relationship between the severity of deterioration of flexible pavements and the walkability. Methodology: It is an applied, non-experimental, descriptive, correlational, cross-sectional research with a qualitative approach; in a town of 150 streets in Nuevo Chimbote. The method for evaluating surface deterioration in flexible pavements and its relationship with passability was used, according to Directorial Resolution No. 022018-MTC / 14. in a sample of 108; The chi square test was used to determine the association between surface deterioration in pavements and walkability. There is a relationship between the severity of surface deterioration and trafficability in flexible pavements at a level of significance of $(P>$ 0.05). Conclusions: The deterioration of flexible pavements yields $25 \%$ good trafficability, $40 \%$ in regular passability and $35 \%$ in poor passability. There is a significant relationship between the deterioration of flexible pavements and walkability.

Keywords: Deterioration of flexible pavements; Walkability. Severity of deterioration of flexible pavement.

\section{INTRODUCCIÓN}

Las vías de comunicación terrestre es la interconexión de actividades económicas, sociales, culturales y comerciales de un pueblo con otro. Verán, (2017), la infraestructura vial terrestre contribuye en el crecimiento económico mediante la interconexión de los mercados y centros de producción. Morales, et al., (2009), comentaron que existen tres clases de pavimentos, flexibles, rígidas y semirrígido; por otro lado, Matos y Núñez (2018: p.16-17), mencionaron que los elementos de la infraestructura vial son la estructura del pavimento, separador central, isletas, rotondas, obras de arte, drenaje, cunetas, muros de contención, taludes y cortes, vehículos, puentes vehiculares y peatonales; rampas peatonales, ciclo-rutas de circulación de bicicleta; seguridad vial. Verán (2017: p.11), indicó que el diseño del pavimento es el conjunto de capas de material seleccionado que recibe de forma directa las cargas del tránsito y las transmiten a los estratos inferiores en forma disipada, proporcionando una superficie de rodamiento. Asimismo, Chirinos (s/f), refirió que el diseño de pavimento tiene en cuenta la subrasante, los materiales, el espesor de las capas, cuando son sometidas las cargas vehiculares sobre la estructura del pavimento, debe ser resistente a la carga vehicular. Monsalve, et al., (2012), identificaron los factores del diseño de pavimentos son la frecuencia de la carga de tránsito vehicular, la máxima presión de contacto, la velocidad de operación del vehículo, y las deformaciones del pavimento. Valle y Yufra (2018), complementaron, en el diseño de pavimento según AASHTO 2008 , las condiciones ambientales de temperatura, humedad, cargas de tránsito, propiedad elástica, espesor de la capa, duración de materiales, análisis de esfuerzos y deformaciones, condición térmica de esfuerzo y deformación. Así mismo Jaña (2016: p.4-22), afirma que la temperatura, la lluvia, la radiación solar, la presión atmosférica, la velocidad del viento y la humedad influyen en deterioros de los pavimentos. Gradilla, et al., (2018), afirma que los impactos climáticos ambientales aceleran el deterioro de las carreteras. Jaña (2016), afirma que el volumen de tránsito, anual, diario y por horas; clase de vehículo; número de carril de dirección, distribución por ejes de carga, número de ejes de camiones, distancia entre ejes; porcentaje de camiones en el carril de diseño; velocidad del vehículo. Pérez, et al., (2019), el desgaste de las capas asfálticas, compactación, deformación de corte, deformación de la subrasante, influyen en la deformación del pavimento. Pérez, et., (2019), la frecuencia de carga vehicular, la deformación permanente al esfuerzo, la densidad del material, la deformación permanente del pavimento se incrementa. Sotil (s/f), la carga de transporte por medio de las llantas se distribuye de la zona de contacto a un área más amplia a través de las capas, causando deformación de la capa baja, que se reflejan en la superficie con fallas

Recibido:27/07/2021 - Aprobado:08/08/2021

Este es un artículo de acceso abierto, distribuido bajo los términos de la Licencia Creative Commons Atribución 4.0 Internacional (http://creativecommons.org/licenses/by/4.0/) 
superficiales. Chang, et al., (2013: p.3-4), la evolución de las fisuras de abajo hacia arriba, según las huellas del neumático a lo ancho del carril de tránsito, causada por cambios bruscos en el gradiente térmico, deformación a nivel de la capa de mezcla asfáltica, base y subrasante. Monsalve, et al., (2012), la fatiga es un estado de tensión provocado por la frecuencia de la carga vehicular, que causa el agotamiento del material, se manifiesta por las fisuras superficiales. Tacza y Rodríguez (2018: p.24-25), las deflexiones causadas por el paso de las ruedas, provocan tensión de tracción en los revestimientos asfalticos, hasta que se rompe con la aparición de fisuras y grietas en pavimentos flexibles. Coy (2017): piel de cocodrilo, agrietamiento en bloque, abultamientos, hundimientos, corrugación, depresión, grieta de borde, grieta de reflexión de junta, desnivel carril / berma, grietas longitudinales y transversales, parcheo en las acometidas de servicios públicos, huecos, ahuellamiento, desplazamiento, desprendimiento de agregados. Uribe (s/f), las fisuras se localizan en el eje de la vía, en el ancho de los carriles, en las zonas de ensanche y zonas de unión de colocación del pavimento asfáltico. Miranda (2010), las fisuras se relacionan con la fatiga de la estructura asfáltica, la pérdida de flexibilidad a temperatura superior $a 30^{\circ} \mathrm{C}$. Uribe (s/f), las fisuras tienden a iniciarse en el fondo de las capas asfálticas, donde los esfuerzos de tracción son mayores bajo la acción de la carga vehicular. Pereda (2014 p. 20), los deterioros progresivos de la superficie de rodadura debido a la carga de tránsito vehicular y los factores climáticos, reducen en la transitabilidad vehicular. Consejo de directores de carreteras de iberia e Iberoamérica (2002), el desprendimiento de agregados en capas de pavimento flexible, cuyas causas pueden ser el esparcido irregular del asfalto, asfalto inadecuado, agregado sucio, insuficiente penetración de imprimación en bases hidráulicas, emulsificado aplicado en caliente, espesor insuficiente de la capa de rodadura, Consejo de directores de carreteras de iberia e Iberoamérica (2002), canalizaciones son deformaciones transversales por hundimiento o elevación de las rodadas, las causas son la capacidad estructural del pavimento insuficiente. Miranda (2010), baches son cavidades formadas al desprenderse la mezcla asfáltica; las causas son estructura inadecuada de la subrasante, defecto constructivo aislado, subdrenaje inadecuado, hundimiento mayor a $2 \mathrm{~cm}$. Miranda (2010), la ondulación es una deformación plástica de la capa asfáltica, debido a una pérdida de estabilidad de la mezcla, agregados redondeados, la mala dosificación del asfalto, uso de ligantes blandos, circulación lenta en pendientes pronunciadas, frenado de vehículos pesados. Miranda (2010), la deformación transversal debido a la estructura insuficiente para el nivel de solicitaciones y características de la subrasante, falta de adherencia entre la carpeta de superficie y la carpeta inferior, drenaje insuficiente, derrame de solventes, la presencia de polvo, aceite o agua entre estas dos carpetas. Uribe (s/f), piel de cocodrilo son fisuras interconectadas, en las zonas de carga vehicular, se propagan formando piezas angulares parecido a la piel de un cocodrilo, de diámetro menor de $30 \mathrm{~cm}$., presenta hundimiento del área afectada; las causas son incompatibilidad de deflexiones con el espesor de la capa de rodadura, subdrenaje inadecuado en sitios aislados, uso de asfaltos muy duros. Uribe (s/f), abultamiento en la superficie del pavimento, ocupando pequeñas áreas, y gradualmente en áreas grandes, acompañados en algunos casos por fisuras. Uribe (s/f), hundimiento son depresiones de diversas formas en el pavimento con respecto al nivel de la rasante, Miranda. (2010), el ahuellamiento es la deformación plástica de la capa del pavimento asfaltico de la trayectoria de las llantas, debido a la fatiga de la estructura ante la repetición de cargas vehiculares. Matos y Núñez (2018), parches por la reparación de servicios de agua, gas, con material insuficiente, deficiencias en las juntas, insuficiente a las características de la subrasante. Transitabilidad, según la Resolución Directoral N 02-2018$\mathrm{MTC} / 14$. Es el nivel de servicio de la infraestructura vial que asegura un estado tal de la misma que permite un flujo vehicular regular durante un determinado periodo, con el criterio de valoración de 50 a 500 puntos.

\section{MATERIAL Y METODOS}

La investigación es no experimental, de enfoque cualitativo, de tipo descriptivo, correlacional, transversal; el objetivo es relacionar la severidad de los deterioros superficiales de pavimentos flexibles en la transitabilidad, El estudio se realizó en la evaluación de los deterioros superficiales de las calles de Nuevo Chimbote durante el año de 2020 de abril a junio. El método de estudio fue visual, toma de datos con GPS, cámara fotográfica y ficha técnica del MTC (2014), como se muestra en la tabla1, así mismo se utilizó flexómetro, cinta métrica, planos de distribución; medición en tramos de 100 metros. La prueba de Chi cuadrado, determinó la asociación de la severidad de deterioro superficial de pavimentos flexibles y la transitabilidad. Para el análisis de los datos se utilizó Excel. El distrito de Nuevo Chimbote pertenece a la provincia de Santa, Región Ancash, republica del Perú; se ubica en la Panamericana Norte Km 426 en las coordenadas geográficas: Latitud: $9^{\circ} 6^{\prime} 56^{\prime \prime}$ Sur; Longitud: $78^{\circ} 31^{\prime} 53^{\prime \prime}$ Oeste; a 22 m.s.n.m. tiene un área de $389.73 \mathrm{Km} 2$. Fue creada con Ley $\mathrm{N}^{\circ}$ 26318 de 27 de mayo de 1994, tiene una población de 151,127 habitantes, con una densidad poblacional de $380.70 \mathrm{hab} / \mathrm{km} 2$ : Las características de la fundación, es una capa de arena limosa de origen eólico, presenta una pendiente moderada, el clima es del tipo desértico con insuficientes precipitaciones pluviales, la temperatura máxima es de $32^{\circ} \mathrm{C}$ en el verano y la mínima de $12^{\circ} \mathrm{C}$ en el invierno; la humedad relativa máxima es de $92 \%$ y la mínima de $72 \%$, presenta vientos constantes durante todo el año, con velocidades de 24 y $30 \mathrm{Km} / \mathrm{h}$. Las características socioeconómicas de la población, un $12 \%$ tienen empleo fijo, un $66 \%$ tiene empleo eventual, y un $13 \%$ son desocupados.

\section{Muestra}

La técnica de recolección de datos de campo fue la auscultación superficial de tipo visual de las fallas superficiales en una población de 150 calles, con tamaño de muestra de 108 , con el $95 \%$ de grados de confianza, 
Tabla 1. Parámetros de severidad de pavimentos flexibles

\begin{tabular}{|c|c|c|}
\hline Deterioro Superficial & Severidad & Índice \\
\hline \multirow[t]{3}{*}{ desprendimiento de capas asfáticas. } & bajo & $<5 \%$ \\
\hline & medio & $5 \%<y<10 \%$ \\
\hline & alto & $10 \%<$ \\
\hline \multirow[t]{3}{*}{ baches en pavimento flexible } & bajo & $1 \%<$ \\
\hline & medio & $1 \% y<10 \%$ \\
\hline & alto & $10 \%<$ \\
\hline \multirow[t]{3}{*}{ ondulaciones en pavimento flexible } & bajo & $6 \mathrm{~m}^{2}$ a $13 \mathrm{~m}^{2}$ \\
\hline & medio & $13 \mathrm{~m}^{2}$ a $25 \mathrm{~m}^{2}$ \\
\hline & alto & $<25 \mathrm{~m}^{2}$ \\
\hline \multirow[t]{3}{*}{ ahuellamiento en pavimento flexible } & bajo & $13 \mathrm{~m}^{2}$ a $25 \mathrm{~m}^{2}$ \\
\hline & medio & $25 \mathrm{~m}^{2}$ a $50 \mathrm{~m}^{2}$ \\
\hline & alto & $<50 \mathrm{~m}^{2}$ \\
\hline \multirow[t]{3}{*}{ fisura longuitudinal en pavimento flexible } & bajo & ancho \\
\hline & medio & ancho \\
\hline & alto & balanceo de carro \\
\hline \multirow[t]{3}{*}{ fisura transversal en pavimento flexible } & bajo & ancho $>1.5 \mathrm{~m}^{2}$ \\
\hline & medio & ancho $>5 \mathrm{~m}^{2}$ \\
\hline & alto & ancho $<5 \mathrm{~m}^{2}$ \\
\hline \multirow[t]{3}{*}{ piel de cocodrilo en pavimento flexible } & bajo & malla $<500 \mathrm{~mm}$ \\
\hline & medio & malla $>500 \mathrm{~mm}$ \\
\hline & alta & malla $<500 \mathrm{~mm}$, con fragmento \\
\hline \multirow[t]{3}{*}{ transitabilidad vehicular } & bueno & $>400$ \\
\hline & regular & $\ldots \ldots \ldots$ \\
\hline & malo & $\leq 150$ \\
\hline
\end{tabular}




\section{RESULTADOS}

Tabla 2. Severidad de deterioro superficial de pavimento flexible en Nuevo Chimbote

\begin{tabular}{|c|c|c|c|c|c|c|c|c|}
\hline \multirow[t]{3}{*}{ Deterioros de pavimentos flexibles } & \multicolumn{8}{|c|}{ severeridad } \\
\hline & \multicolumn{2}{|c|}{ bajo } & \multicolumn{2}{|c|}{ medio } & \multicolumn{2}{|c|}{ alto } & \multicolumn{2}{|l|}{ total } \\
\hline & $\mathrm{N}^{\circ}$ & $\%$ & $\mathrm{~N}^{\circ}$ & $\%$ & $\mathrm{~N}^{\circ}$ & $\%$ & $\mathrm{~N}^{\circ}$ & $\%$ \\
\hline desprendimiento de capas asfálticas. & 41 & 38 & 45 & 30 & 34 & 32 & 108 & 100 \\
\hline baches en pavimento flexible. & 43 & 40 & 43 & 40 & 22 & 20 & 108 & 100 \\
\hline ondulaciones en pavimento flexible. & 59 & 55 & 22 & 20 & 27 & 25 & 108 & 100 \\
\hline ahuellamiento en oavimento flexible. & 54 & 50 & 32 & 22 & 30 & 20 & 108 & 100 \\
\hline fisura longuitudinal en pavimento flexible & 49 & 45 & 27 & 25 & 32 & 30 & 108 & 100 \\
\hline fisura transversal en pavimento flexible & 41 & 38 & 32 & 30 & 35 & 32 & 108 & 100 \\
\hline piel de cocodrilo en pavimento flexible & 48 & 44 & 28 & 26 & 32 & 30 & 108 & 100 \\
\hline
\end{tabular}

Tabla 3. Transitabilidad de las calles con pavimentos flexibles.

\begin{tabular}{|l|l|l|l|l|l|l|l|l|}
\hline \multirow{2}{*}{ Condición de la calles } & \multicolumn{6}{l|}{ Estado de pavimento flexibles } \\
\cline { 2 - 10 } & \multicolumn{2}{|l|}{ bueno } & \multicolumn{2}{l|}{ regular } & \multicolumn{2}{l|}{ malo } & \multicolumn{2}{l|}{ total } \\
\cline { 2 - 10 } & $\mathrm{N}^{\circ}$ & $\%$ & $\mathrm{~N}^{\circ}$ & $\%$ & $\mathrm{~N}^{\circ}$ & $\%$ & $\mathrm{~N}^{\circ}$ & $\%$ \\
\hline transitabilidad & 27 & 25 & 43 & 40 & 38 & 35 & 108 & 100 \\
\hline
\end{tabular}

En la tabla 2, se observa, de 108 muestras, un $38 \%$ de severidad de desprendimientos de capas asfálticas es bajo, un $30 \%$ de severidad de desprendimientos de capas asfálticas es medio y un $32 \%$ de severidad de desprendimientos de capas asfálticas es alto. Un $40 \%$ de severidad de baches es bajo, un $40 \%$ de severidad de baches es medio y un $20 \%$ de severidad de baches es alto en pavimentos flexibles. Un $55 \%$ de severidad de ondulación es bajo, un $20 \%$ de severidad de ondulación es medio y un $25 \%$ de severidad de ondulación es alto en pavimento flexible. Un $50 \%$ de severidad de ahuellamiento es bajo, un $30 \%$ de severidad de ahuellamiento es alto y un $30 \%$ de severidad de ahuellamiento es alto en pavimentos flexibles. Un $45 \%$ de severidad de fisura longitudinal es bajo, un $25 \%$ de severidad de fisura longitudinal es medio y un $30 \%$ de severidad de fisura longitudinal es alto en pavimentos flexibles. Un $38 \%$ de severidad de fisura transversal es bajo, un $30 \%$ de severidad de fisura transversal es medio y un $32 \%$ de severidad de fisura transversal es alto en pavimentos flexibles. Un $44 \%$ de severidad de piel de cocodrilo es bajo, un $26 \%$ de severidad de piel de cocodrilo es medio y un $30 \%$ de severidad de piel de cocodrilo es alto en pavimentos flexibles, tal como se muestra en la tabla 2 . Se ha determinado un $25 \%$ de transitabilidad en buenas condiciones, un $40 \%$ de transitabilidad en regular condición y un 35\% de transitabilidad en malas condiciones, tal como se muestra en la tabla 3.

\section{DISCUSIÓN}

Monsalve, et al., (2012), la frecuencia de tránsito vehicular, la presión de contacto, la velocidad del vehículo deforma el pavimento flexible. Valle y Yufra (2018), la temperatura, humedad, Jaña (2016), la lluvia, la radiación solar, la velocidad del viento y la humedad influyen en deterioros de pavimentos. Sotil (s/f), las llantas se distribuyen en la zona de contacto causando fallas superficiales. Chang, et al (2013), las fisuras según las huellas del neumático deforman la capa asfáltica. Tacza y Rodríguez (2018), el paso de las ruedas provoca tensión de tracción hasta la aparición de fisuras y grietas. Coy (2017), como piel de cocodrilo, agrietamiento en bloque, 
hundimientos, corrugación, depresión, grieta de borde, desnivel carril /berma, grietas longitudinales y transversales, parcheo en las acometidas de servicios públicos, ahuellamiento, desprendimiento de agregados. Pereda (2014), la carga de tránsito vehicular y factores climáticos deterioran la superficie de rodadura y reducen la transitabilidad. La transitabilidad en pavimentos flexibles de Nuevo Chimbote se halló: un $25 \%$ bajo, un $40 \%$ medio y un $35 \%$ alto.

Hipótesis especifica 1.

HO: No existe relación entre la severidad de los desprendimientos de capas asfálticas y la transitabilidad.

$\mathrm{H} 1$ : Existe relación entre la severidad de los desprendimientos de capas asfálticas y la transitabilidad.

Prueba Chi cuadrado entre severidad de desprendimientos de capas asfálticas y transitabilidad. Nivel de significancia $=0.05$

Estadístico de prueba

\begin{tabular}{|r|l|}
\hline Chi cuadrado calculado & $p$ \\
\hline 23,87 & $0,0000^{*}$ \\
\hline
\end{tabular}

${ }^{*} \mathrm{P}=0,000<0,05$ significativo

Regla de decisión: Rechazar Ho si Chi cuadrado calculado $>$ Chi tabla $(2)(2) 0,05=$

Chi tabla (4)0.,5=9,48

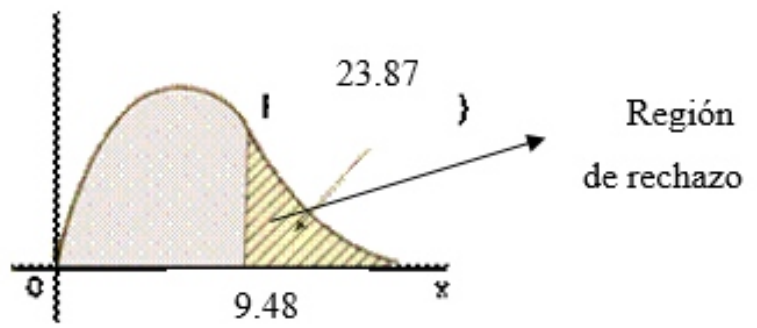

Dado que Chi cuadrado: $23,87>=9,48$ entonces rechazar Ho, es decir. Existe relación entre la severidad de desprendimientos de capas asfálticas y la transitabilidad.

\section{Hipótesis específica 2}

HO: No existe relación entre la severidad de baches en pavimentos flexibles y la transitabilidad.

$\mathrm{H} 1$ : Existe relación entre la severidad de baches en pavimentos flexibles y la transitabilidad.

Nivel de significancia 0,05

Estadístico de prueba

\begin{tabular}{|l|l|}
\hline Chi cuadrado calculado & $\mathrm{p}$ \\
\hline 54,2 & $0,0000^{*}$ \\
\hline
\end{tabular}

${ }^{*} \mathrm{P}=0.000<0.05$ significativo

Regla de decisión: Rechazar Ho si Chi cuadrado calculado $>$ Chi tabla (2) (2)0,05=

Chi tabla $(4) 0,05=9,4$

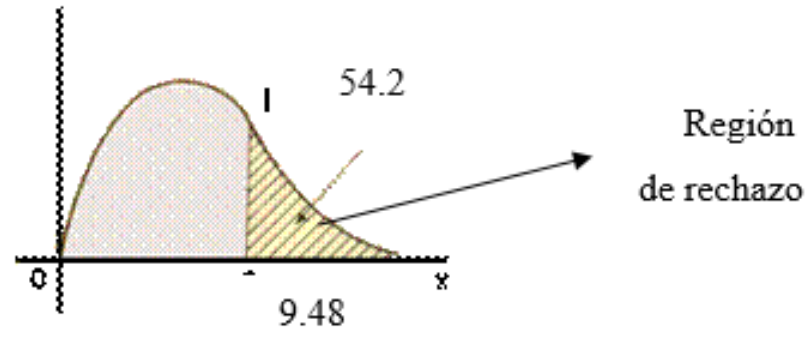

Dado que Chi cuadrado calculado: $54.2>=9.48$ entonces rechazar Ho, es decir. Existe relación entre la severidad de baches en pavimentos flexibles y la transitabilidad.

\section{Hipótesis específica 3}

HO: No existe relación entre la severidad de ondulaciones en pavimentos flexibles y transitabilidad.

H1: Existe relación entre la severidad de ondulaciones en pavimentos flexibles y la transitabilidad.

Nivel de significancia 0.05

Estadístico de prueba 8

\begin{tabular}{|r|l|}
\hline \multicolumn{1}{l|}{ Chi cuadrado calculado } & $\mathrm{p}$ \\
\hline 21,3 & $0,0000^{\star}$ \\
\hline
\end{tabular}

${ }^{*} P=0.000<0,05$ significativo

Regla de decisión: Rechazar Ho si Chi cuadrado calculado $>$ Chi tabla $(2)(2) 0,05=$

Chi tabla (4)0,05 $=9,48$

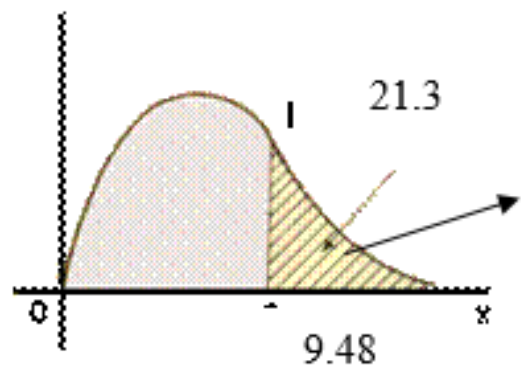

\section{Región}

de rechazo

Dado que Chi cuadrado: 21,3 >=9,48 entonces rechazar Ho, es decir. Existe relación entre la severidad de ondulaciones en pavimentos flexibles y la transitabilidad.

\section{Hipótesis específica 4}

HO: No existe relación entre la severidad de ahuellamientos en pavimentos flexibles y transitabilidad.

H1: Existe relación entre la severidad de ahuellamientos en pavimentos flexibles y la transitabilidad.

Nivel de significación 0,05

Estadístico de prueba 


\begin{tabular}{|r|l|}
\hline Chi cuadrado calculado & $p$ \\
\hline 15,3 & $0,0000^{*}$ \\
\hline
\end{tabular}

${ }^{*} P=0,000<0,05$ significancia

Regla de decisión: Rechazar Ho si Chi cuadrado calculado $>$ Chi tabla (2)(2)0,05=

Chi tabla $(4) 0,05=9,48$

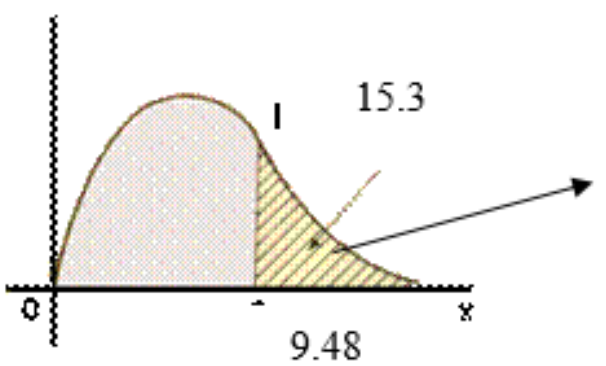

\section{Región}

de rechazo

Dado que Chi cuadrado: 15,3 >=9,8 entonces rechazar Ho, es decir. Existe relación entre la severidad de ahuellamientos en pavimentos flexibles y la transitabilidad.

Hipótesis específica 5

$\mathrm{HO}$ : No existe relación entre la severidad de fisuras longitudinales en pavimentos flexible y la transitabilidad.

$H 1$ : Existe relación entre la severidad de fisuras longitudinales en pavimentos flexibles y la transitabilidad.

Nivel de significancia 0,05

Estadístico de prueba

\begin{tabular}{|r|l|}
\hline Chi cuadrado calculado & $\mathrm{p}$ \\
\hline 17,5 & $0,0000^{*}$ \\
\hline
\end{tabular}

${ }^{*} \mathrm{P}=0,000<0,05$ significativo

Regla de decisión: Rechazar Ho si Chi cuadrado calculado $>$ Chi tabla (2)(2)0,05= Chi tabla (4)0,05=9,48

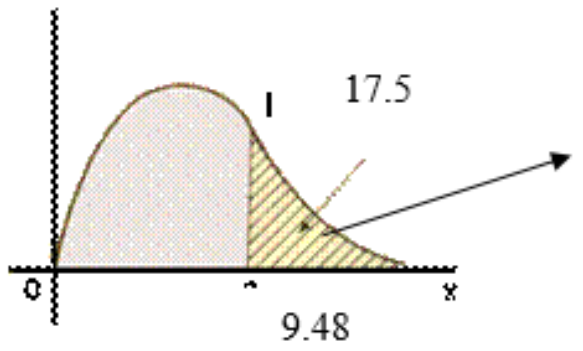

Región de rechazo

Dado que Chi cuadrado: 17,5 >=9,48 entonces rechazar Ho, es decir. Existe relación entre la severidad de fisuras longitudinales en pavimentos flexibles y la transitabilidad.

Hipótesis específica 6

HO: No existe relación entre la severidad de fisuras transversales y la transitabilidad.

H1: Existe relación entre la severidad de fisuras transversales y la transitabilidad.

Nivel de significancia 0,05

Estadístico de prueba

\begin{tabular}{|l|l|}
\hline Chi cuadrado calculado & $p$ \\
\hline 27,3 & $0,0000^{*}$ \\
\hline
\end{tabular}

${ }^{*} \mathrm{P}=0.000<0.05$ significativo

Regla de decisión: Rechazar Ho si Chi cuadrado calculado $>$ Chi tabla (2)(2)0,05= Chi tabla (4)0,05=9,48

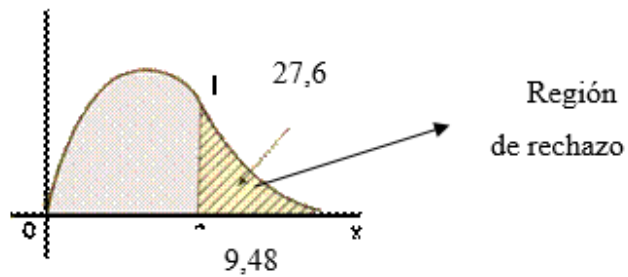

Dado que Chi cuadrado: 27,6>=9,48 entonces rechazar Ho, es decir. Existe relación entre la severidad de fisuras transversales en pavimentos flexibles y la transitabilidad.

Hipótesis específica 7

HO: No existe relación entre la severidad de piel de cocodrilo en pavimentos flexibles y la transitabilidad.

H1: Existe relación entre la severidad de piel de cocodrilo en pavimentos flexibles y la transitabilidad.

Nivel de significancia 0,05

Estadístico de prueba

\begin{tabular}{|r|l|}
\hline Chi cuadrado calculado & $p$ \\
\hline 30,2 & $0,0000^{*}$ \\
\hline
\end{tabular}

${ }^{*} \mathrm{P}=0,000<0,05$ significativo

Regla de decisión: Rechazar Ho si Chi cuadrado calculado $>$ Chi tabla $(2)(2) 0,05=$ Chi tabla $(4) 0,05=9,48$

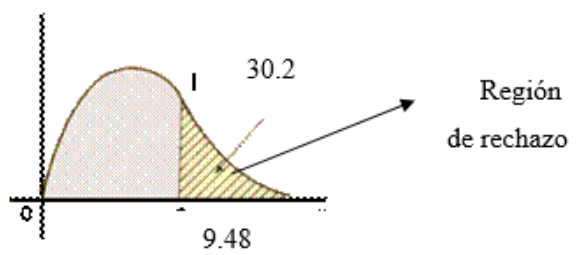

Dado que Chi cuadrado: 30,2>=9,48 entonces rechazar Ho, es decir. Existe relación entre la severidad de piel de cocodrilo en pavimentos flexibles y la transitabilidad. 


\section{CONCLUSIONES}

Los deterioros de los pavimentos flexible de Nuevo Chimbote son:

Un $25 \%$ de los deterioros de los pavimentos flexibles son de buena transitabilidad.

Un $40 \%$ de los deterioros de los pavimentos flexibles son de regular transitabilidad.

Un $35 \%$ de los deterioros de los pavimentos flexibles son de mala transitabilidad.

Con la prueba de chi cuadrado $\mathrm{P}=0.000<0,05$ significativo, se demostró que. Existe relación entre la severidad de deterioros superficiales en pavimentos flexibles en la transitabilidad en las calles de Nuevo Chimbote.

\section{REFERENCIAS BIBLIOGRÁFICAS}

Consejo de directores de carreteras de iberia e Iberoamérica. M5.1. (2002). Catálogo de deterioros de pavimentos flexibles. Colección de documento. volumen n 11. México. https://docplayer.es/5535890-M5-1-catalogode-deterioros-de-pavimentos-flexibles.html

Coy, O. M. (2017). Evaluación superficial de un pavimento flexible de la calle 134 entre carreras $52^{a}$ a $53 c$ comparando los métodos VIZIR y PCI. [Tesis de especialización en ingeniería civil, Universidad Militar Nueva Granada]. https://repository.unimilitar.edu.co/handle/10654/16508.

Chang, C. M., Vidal, J., Loria, L. G., Bustos, M., Delgadillo, R. (2013). Aplicabilidad del método mecanístico empírico de diseño de pavimentos (MEPDG) AASHTO 2008 en Latinoamérica. [Guía IAG239-03-2013, Universidad de Texas a t E I p a so E. U. A ] . https://www.lanamme.ucr.ac.cr/repositorio/handle/50625 $112500 / 808$

Chirinos, E. (s/f). Métodos de diseño de pavimentos. [PPT, Universidad experimental Francisco de Miranda]. https://www.slideshare.net/eliezerchirinos/mtodos-dediseo-de-pavimentos?qid=7372e46e-

Jaña, C. G. (2016). Implementación de la guía de diseño mecanístico-empírico

AASHTO 2008 en la región Piura. [Tesis de grado, Universidad de Piura]. https://pirhua.udep.edu.pe/handle/11042/3143

Gradilla, L. A., Mendoza, J. F., Orantes, H., Marcos, O. A. (2018). Aproximación geoespacial para la adaptación al cambio climático de la infraestructura carretera en México. [Publicación Técnica $N^{\circ}$ 523, Instituto mexicano de

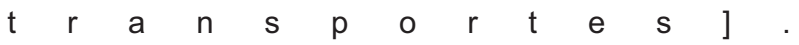
https://imt.mx/archivos/Publicaciones/PublicacionTecnic a/pt523.pdf

Matos, D. R. y Núñez, F. B. (2018). Evaluación del sistema de gestión de pavimentos de la carretera Central (tramo: la Oroya - Concepción "PE003-S") [Tesis de pregrado, Universidad peruana de ciencias aplicadas]. https://repositorioacademico.upc.edu.pe/handle/10757/6 24119.

Miranda, R. J. (2010). Deterioro de pavimentos flexibles y rígidos [Tesis de pregrado, Universidad Austral de Chile]. https://docplayer.es/13234356-Deterioros-enpavimentos-flexibles-y-rigidos.html

Monsalve, L. M., Giraldo, L. C., Maya, J. Guzmán. M. R. (2012). Diseño de pavimento flexible y rígido [Universidad de Quindio]. https://www.slideshare.net/rosabeatrizvillaloboshuaman/ diseo-de-pavimento-flexible-y-rgido?next_slideshow=1.

Pereda, C. V. (2014). Índice de condición de pavimento de la carretera Cajamarca - la Colpa. [tesis de pregrado, Universidad Nacional de Cajamarca]. https://repositorio.unc.edu.pe/bitstream/handle/UNC/498 /T\%20625.8\%20P434\%202014.pdf?sequence=1\&isAllo wed $=y$

Pérez, N., Garnica, P., García, L. A., García, A. (2019). Evaluación de la deformación permanente en una trayectoria de humedecimiento o secado. [Publicación técnica $n^{\circ} 561$, Instituto mexicano de transportes]. https://imt.mx/archivos/Publicaciones/PublicacionTecnic a/pt561.pdf

Resolución Directoral N 02-2018-MTC/14. [Glosario de $\mathrm{t}$ é $\mathrm{r} \mathrm{m}$ i $\mathrm{n}$ o s, M T C ]. https://www.gob.pe/institucion/mtc/normaslegales/10338-002-2018-mtc-14

Sotil, A. Pavimentos. [Tema de trabajo, Universidad $\left.\begin{array}{llllllllllll}C & o & n & t & i & n & e & n & t & a & l & \end{array}\right]$. https://www.slideshare.net/kevinromerolatorre/tipos-depavimentos.

Tacza, E. B., Rodríguez, B. O. (2018). Evaluación de fallas mediante el método $\mathrm{PCl}$ y planteamiento de alternativas de investigación para mejorar la condición operacional del pavimento flexible en el carril segregado del corredor Javier Prado. [Tesis de grado, Universidad Peruana de C i encias A p I i c a d a s ]. https://repositorioacademico.upc.edu.pe/handle/10757/6 24556

Uribe, EJ. Análisis del comportamiento de la carpeta asfáltica en el puente el cortijo ubicado en la ciudad de Bogotá D.C. Colo m bia. Tesis de grado. https://repository.unimilitar.edu.co/handle/10654/12164

Valle, LE. Yufra, JR. Implementación del modelo climático del método AASHTO 2008 (MEPDG) para el diseño de pavimentos flexibles en la ciudad de Tacna. Tesis de $\mathrm{g} \mathrm{r}$ a d o. 2018. http://repositorio.upt.edu.pe/handle/UPT/603

Verán, D. (2017). Evaluación ambiental de un tramo especifico de la autopista Panamericana Sur, usando la metodología de análisis de ciclo de vida [Tesis de pregrado, Universidad peruana de ciencias aplicadas]. file:///C:/Users/User/Downloads/VERAN_DANIEL_AUT OPISTA_ANALISIS_CICLO_VIDA\%20(2).pdf 